\title{
Geometric Models of the Relativistic Harmonic Oscillator
}

\author{
Ion I. Cotăescu \\ The West University of Timişoara, \\ V. Pârvan Ave. 4, RO-1900 Timişoara, Romania
}

June 24, 2021

\begin{abstract}
A family of relativistic geometric models is defined as a generalization of the actual anti-de Sitter $(1+1)$ model of the relativistic harmonic oscillator. It is shown that all these models lead to the usual harmonic oscillator in the non-relativistic limit, even though their relativistic behavior is quite different. Among quantum models we find a set of models with countable energy spectra, and another one having only a finite number of energy levels and in addition a continuous spectrum.
\end{abstract}

The problem of the relativistic generalization of the (classical or quantum) non-relativistic harmonic oscillator (NRHO) has been frequently discussed, but as yet there is no unique definition of the relativistic harmonic oscillator (RHO). In the context of general relativity, the RHO has been defined as a free system on the anti-de Sitter (AdS) static background. There are many phenomenologic [1, 2] and group theoretic [3, [, 5] arguments for this geometric model. Its advantage is that the constants of the classical motion (on geodesics) satisfy the so $(1,2)$ algebra (of the AdS symmetry [3]), realized by Poison brackets for any AdS metric. However, the concrete choice of the metric is also important from the observer's point of view because the form of the classical trajectory, as well as the modes of the corresponding quantum 
system, depend on it. For the $(1+1)$ RHO the $(1+3)$ metric proposed in Ref. [2] gives the following $(1+1)$ AdS metric

$$
d s^{2}=\frac{1}{1-\omega^{2} x^{2}} d t^{2}-\frac{1}{\left(1-\omega^{2} x^{2}\right)^{2}} d x^{2}
$$

which reproduces the classical non-relativistic equation of motion, i.e. $\ddot{x}+$ $\omega^{2} x=0$. The corresponding quantum system has been analyzed in Ref. [6], starting with another AdS metric which can be derived from (11) by changing the space coordinate $x \rightarrow x^{\prime}=x / \sqrt{1+\omega^{2} x^{2}}$. The result is an equidistant energy spectrum with a groundstate energy larger than, but approaching $\omega / 2$ in the non-relativistic limit (in natural units, $\hbar=c=1$ ). Since, partularlly, the space coordinate transformations of the static backgrounds do not change the quantum modes, we can say that the RHO is well simulated by the free motion on the AdS background with the metric (1).

However, one can ask if there are more geometric models which should behave as the NRHO in the non-relativistic limit. In order to give an answer, we shall study here the family of models having the metrics

$$
d s^{2}=g_{00} d t^{2}+g_{11} d x^{2}=\frac{1+(1+\lambda) \omega^{2} x^{2}}{1+\lambda \omega^{2} x^{2}} d t^{2}-\frac{1+(1+\lambda) \omega^{2} x^{2}}{\left(1+\lambda \omega^{2} x^{2}\right)^{2}} d x^{2},
$$

where $\lambda$ is a real parameter. Our aim is to investigate both the classical and the quantum free motions of a particle of mass $m$, and to show that all these models lead to the NRHO in the non-relativistic limit. Moreover, we shall try to point out their specific relativistic effects.

The metrics (2) represent a generalization of the AdS metric (1). In fact these are conformal transformations depending on $\lambda$ of some AdS metrics if $\lambda<0$, of some static de Sitter metrics if $\lambda>0$, or of the the Minkowski flat metric when $\lambda=0$. The parameterization of these transformations has been defined in a such a manner to obtain the exact AdS metric (11) for $\lambda=-1$. We note that the event horizon of an observer situated at $x=0$ is at $R_{+}=\infty$ for $\lambda \geq 0$ and at $R_{-}=1 / \omega \sqrt{-\lambda}$ in the case of $\lambda<0$ in which the metrics have singularities. This will give the space domain of the free motion, $D=(-R, R)$.

First, we shall derive the classical equation of motion starting with the geodesics equation, (non-covariantly) expressed in terms of $x(t)$ and its time 
derivatives $\dot{x}$ and $\ddot{x}$,

$$
\ddot{x}-\frac{g_{00, x}}{2 g_{11}}+(\dot{x})^{2}\left(\frac{g_{11, x}}{2 g_{11}}-\frac{g_{00, x}}{g_{00}}\right)=0 .
$$

In addition we shall use the conservation of the energy $E$ (on the static backgrounds), which gives

$$
(\dot{x})^{2}=\frac{g_{00}}{g_{11}}\left(\frac{m^{2} g_{00}}{E^{2}}-1\right)
$$

From (2), (3) and (4) we obtain

$$
\ddot{x}+\Omega^{2} x=0,
$$

where

$$
\Omega=\frac{\omega}{E} \sqrt{(1+\lambda) m^{2}-\lambda E^{2}}
$$

is the effective frequency. Its dependence on energy can be considered as a pure relativistic effect. We note that $\Omega$ does not depend on $E$ only in the case of the AdS metric when $\lambda=-1$. The trajectory,

$$
x(t)=a \sin \left(\Omega\left(t-t_{0}\right)\right),
$$

is one of oscillations if $\Omega$ and the amplitude,

$$
a=\frac{1}{\omega}\left(\frac{E^{2}-m^{2}}{(1+\lambda) m^{2}-\lambda E^{2}}\right)^{\frac{1}{2}}
$$

are real numbers. We observe that for $\lambda>0$ and $E^{2} \geq m^{2}(1+1 / \lambda)$ these oscillations degenerate into open (uniform or accelerated) motions on $D=(-\infty, \infty)$. Hence, for $\lambda>0$ the system oscillates only for $E \in$ $[m, m \sqrt{1+1 / \lambda})$. This could lead to a finite discrete energy spectrum for the quantum motion. However, when $\lambda<0$ the system oscillates for all the possible energies, $E \in[m, \infty)$, with amplitudes remaining less than $R_{-}$. Now, we can verify that in the non-relativistic limit, for very small $E_{n r}=E-m$ and for any $\lambda$, we obtain the familiar expressions $\Omega \rightarrow \omega$ and $a^{2} \rightarrow 2 E_{n r} / m^{2} \omega^{2}$. Therefore, at least in the case of the classical motion, all these models have 
as the non-relativistic limit the classical NRHO. It remains to verify if this property remains valid also for the quantum motion.

The quantum free motion of a spinless particle is described by the scalar field $\phi$ defined on $D$ minimally coupled with the gravitational field [7] given by the metric (2). Because of energy conservation, the Klein-Gordon equation

$$
\frac{1}{\sqrt{-g}} \partial_{\mu}\left(\sqrt{-g} g^{\mu \nu} \partial_{\nu} \phi\right)+m^{2} \phi=0
$$

where $g=\operatorname{det}\left(g_{\mu \nu}\right)$, admits a set of fundamental solutions (of positive and negative frequency) of the form

$$
\phi_{E}^{(+)}=\frac{1}{\sqrt{2 E}} e^{-i E t} U_{E}(x), \quad \phi^{(-)}=\left(\phi^{(+)}\right)^{*},
$$

which must be orthogonal with respect to the relativistic scalar product [7]

$$
<\phi, \phi^{\prime}>=i \int_{D} d x \sqrt{-g} g^{00} \phi^{*} \stackrel{\leftrightarrow}{\partial_{0}} \phi^{\prime}
$$

Starting with the metric (2) we obtain the Klein-Gordon equation

$$
\left(1+\lambda \omega^{2} x^{2}\right) U_{, x x}+\lambda \omega^{2} x U_{, x}+E^{2} U-\frac{\left(1+(1+\lambda) \omega^{2} x^{2}\right)}{\left(1+\lambda \omega^{2} x^{2}\right)} m^{2} U=0
$$

and the concrete form of the scalar product

$$
<U, U^{\prime}>=\int_{D} \frac{d x}{\sqrt{1+\lambda \omega^{2} x^{2}}} U^{*} U^{\prime}
$$

In the following we shall try to derive the energy spectrum and the form of the wave functions up to normalization factors.

When $\lambda=0$ the equation (12) becomes

$$
-U_{n, x x}+m^{2} \omega^{2} x^{2} U_{n}=\left(E_{n}^{2}-m^{2}\right) U_{n}
$$

from which it results that the wave functions $U_{n}$ coincide with those of the NRHO, while the energy spectrum

$$
E_{n}^{2}=m^{2}+2 m \omega\left(n+\frac{1}{2}\right), \quad n=0,1,2, \ldots
$$


goes to the traditional one in the non-relativistic limit.

In the general case of any $\lambda \neq 0$ it is convenient to use the new variable $y=-\lambda \omega^{2} x^{2}$, and the notations

$$
\epsilon=\frac{E}{\lambda \omega}, \quad \mu=\frac{m}{\lambda \omega}, \quad \nu=\frac{1}{4}\left[(1+\lambda) \mu^{2}-\lambda \epsilon^{2}\right] .
$$

We shall look for a solution of the form

$$
U(y)=N(1-y)^{p} y^{s} F(y),
$$

where $p$ and $s$ are real numbers and $N$ is the normalization factor. After a few manipulation we find that, for

$$
s(2 s-1)=0, \quad 4 p^{2}-2 p-\mu^{2}=0,
$$

the equation (12) transforms into the following hypergeometric equation:

$$
y(1-y) F_{, y y}+\left[2 s+\frac{1}{2}-y(2 p+2 s+1)\right] F_{, y}-\left[(p+s)^{2}-\nu\right] F=0 .
$$

This has the solution 8

$$
F=F\left(p+s-\sqrt{\nu}, p+s+\sqrt{\nu}, 2 s+\frac{1}{2}, y\right),
$$

which depends on the possible values of the parameters $p$ and $s$. From (18) it follows that

$$
s=0, \frac{1}{2}, \quad p=p_{ \pm}=\frac{1}{4}\left[1 \pm \sqrt{1+4 \mu^{2}}\right] .
$$

Moreover, when

$$
\nu=\left(p+s+n^{\prime}\right)^{2}, \quad n^{\prime}=0,1,2 \ldots,
$$

$F$ reduces to a polynomial of degree $n^{\prime}$ in $y$. By using these results, we can establish the general form of the solutions of (12), which could be square integrable with respect to (13), namely

$$
U_{n^{\prime}, s}(x)=N_{n^{\prime}, s}\left(1+\lambda \omega^{2} x^{2}\right)^{p} x^{2 s} F\left(-n^{\prime}, 2 p+2 s+n^{\prime}, 2 s+\frac{1}{2},-\lambda \omega^{2} x^{2}\right) .
$$

Furthermore, we shall define the quantum number $n=2\left(n^{\prime}+s\right)$ which has odd values for $s=0$ and even values for $s=1 / 2$. For both sequences, (22), (18) and (16) give the same formula of the energy levels,

$$
E_{n}{ }^{2}=m^{2}-\lambda \omega^{2}\left[4 p\left(n+\frac{1}{2}\right)+n^{2}\right], \quad n=0,1,2 \ldots
$$


Now, it remains to fix the suitable values of $p$ for which $<U_{n^{\prime}, s}, U_{n^{\prime}, s}><\infty$, and to analyze the structure of the obtained spectra.

Let us first take $\lambda>0$. In this case $D=(-\infty, \infty)$, and the solution (23) will be square integrable only if $p=p_{-}$and $n<-2 p_{-}$. This means that the discrete spectrum is finite, with $n=0,1,2 \ldots n_{\max }$, where $n_{\max }$ is the integer part of $\left(\sqrt{1+4 \mu^{2}}-1\right) / 2$. One can verify that this discrete spectrum is included in the domain of energies $[m, m \sqrt{1+1 / \lambda})$, for which the classical motion is oscillatory. On the other hand, when $E>m \sqrt{1+1 / \lambda}$, then $\nu$ is negative and the hypergeometric functions (20) cannot be reduced to polynomials, but remain analytic for negative arguments. Therefore the functions

$$
U_{\nu, s}=N_{\nu, s}\left(1+\lambda \omega^{2} x^{2}\right)^{p_{-}} x^{2 s} F\left(p_{-}+s-\sqrt{\nu}, p_{-}+s+\sqrt{\nu}, 2 s+\frac{1}{2},-\lambda \omega^{2} x^{2}\right)
$$

can be interpreted as the non-square integrable solutions corresponding to the continuous energy spectrum $[m \sqrt{1+1 / \lambda}, \infty)$.

In the case of $\lambda<0$ the domain $D=\left(-R_{-}, R_{-}\right)$is finite (as in Ref. [2]) and, therefore, the polynomial solutions (23) will be square integrable over $D$ only if $p=p_{+}$. We observe that there are no restrictions on the range of $n$ and, consequently, the discrete spectrum will be countable. Moreover, in this case we have no continuous spectrum since the hypergeometric functions (20) generally diverge for $y \rightarrow 1$ (when $x \rightarrow R_{-}$). We note that for the AdS metric $(\lambda=-1)$ we obtain the same result as in Ref. [6], namely $E_{n}=\omega\left(2 p_{+}+n\right)$.

Thus we have solved the quantum problem for all the values of the parameter $\lambda$. Herein it is interesting to observe that our results are continuous in $\lambda$. More precisely, in the limit of $\lambda \rightarrow 0$ the general formulae (24) and (23) will give the energy spectrum (15) and the NRHO wave functions. Indeed, we observe that, in this limit, $p \sim-m / 2 \lambda \omega$ (since we have choose $p=p_{-}<0$ for $\lambda>0$ and $p=p_{+}>0$ for $\lambda<0$ ) and $n_{\max } \sim m / \lambda \omega \rightarrow \infty$. Therefore, the finite discrete spectra of the models with $\lambda>0$ become countable while the continuous spectra disappear. Hence, it is obvious that all the discrete spectra given by (24) go to (15) when $\lambda \rightarrow 0$. Furthermore, we can calculate, up to factors, the limit of the wave functions (23). We obtain

$$
U_{n^{\prime}, s} \rightarrow \sim e^{-m \omega x^{2} / 2} x^{2 s} F\left(-n^{\prime}, 2 s+\frac{1}{2}, m \omega x^{2}\right) \sim e^{-m \omega x^{2} / 2} H_{n}(\sqrt{m \omega} x),
$$


where $H_{n}$ are Hermite polynomials and $n=2\left(n^{\prime}+s\right)$ as defined above. Thus it results that all the functions (23) go to the specific NRHO wave function which are just the wave functions for $\lambda=0$. For this reason the hypergeometric functions of (23) with the factors $x^{2 s}$ could be considered as a generalization of the Hermite polynomials.

The non-relativistic limit of our models, defined by $m / \omega \rightarrow \infty$, can be easily calculated starting with the observation that, according to (16) and (21), this is equivalent with the limit $\lambda \rightarrow 0$ and, in addition, $m \gg \omega$. Hence, the non-relativistic limit of a model with any $\lambda$ will be the same as in the case of $\lambda=0$, i.e. the NRHO. Therefore, we can conclude that all the models of RHO we have studied here have the same non-relativistic limit, even though they are in fact very different.

Finally we must specify that among these models only one has the whole properties of the NRHO. This is that of the AdS metric for which: i. the classical motion is oscillatory with a fixed frequency (independent on $E$ ), and, ii. the quantum energy spectrum is equidistant. Another interesting model is that of $\lambda=0$ because its wave functions coincide with those of NRHO. However, in general, the models with $\lambda \neq-1$ can not be considered as pure harmonic oscillators since these have not the above mentioned properties of the AdS model. On the other hand, the new models we have considered are interesting because their specific countable or finite discrete energy spectra could allow to identify new observable relativistic effects.

\section{References}

[1] E. van Beveren, G. Rupp, T. A. Rijken, C. Dullemond, Phys. Rev. D 27, 1527 (1983)

[2] C. Dullemond, E. van Beveren, Phys. Rev. D 28, 1028 (1983)

[3] V. Aldaya, J. Bisquert, J. Navarro-Salas, Phys. Lett. A156, 315 (1991)

[4] V. Aldaya. J. A. de Azcarraga, J. Math. Phys. 23, 1297 (1982)

[5] V. Aldaya, J. Bisquert, R. Loll, J. Navarro-Salas, J. Math. Phys. 33, 3087 (1992)

[6] D. J. Navarro, J. Navarro-Salas, preprint HEP-TH-9406001 
[7] N. D, Birrel, P. C. W. Davies, Quantum Fields in Curved Space, Cambridge University Press, Cambridge (1982)

[8] M. Abramowitz, I. A. Stegun, Handbook of Mathematical Functions, Dover, New York (1964) 